\title{
Revival of a non-deforming apicolytic thoracoplasty for the treatment of pulmonary tuberculosis
}

\author{
Antonio Salas ${ }^{1,2}$ and Alfonso Delgado ${ }^{*}$ \\ ${ }^{1}$ Chihuahua University Faculty of Medicine and Biomedical Sciences, Chihuahua, Chih, México \\ ${ }^{2}$ Department of Thoracic Surgery, IMSS General Hospital, Chihuahua, Chih, México
}

\begin{abstract}
The emergence of multidrug-resistant pulmonary tuberculosis (MDR-PTB) poses a serious challenge to the global control of TB. The majority of new cases and deaths occur in developing countries, mainly due to severe problems to ensure proper treatment and to complicating factors such as the AIDS/HIV pandemic; even under these circumstances, phthisiosurgery remains as a controversial procedure for the treatment of TB. We report a reliable, non-deforming, foreign materials-free phthisiosurgery technique for the treatment of complicated, treatment failure and MDR PTB.
\end{abstract}

\section{Introduction}

Before anti-tuberculosis drugs, collapse therapy was a well-established surgical treatment for TB. The methods and their combinations ranged from artificial pneumothorax and pneumoperitoneum to thoracoplasty and plombage [1]. These methods demanded an extensive experience to establish the indication criteria, and were known to have short- and long-term complications. Postoperative problems were more common in the plombage approach because the foreign materials might infect, migrate, irritate or compress other structures. However, the repeated appearances of phthisiosurgery sequels [2-5] speak on behalf the significant successes these old procedures had; moreover, even if not fully rehabilitated phthisiosurgery patients were rendered noninfectious.

To deal with the complications of collapse therapy and to overcome the disadvantages inherent to rib resection, surgeons gradually developed the Apicolytic and the Osteoplastic techniques to Thoracoplasty [6-8]. Here, we present a re- adaptation of these techniques into one that has been named: "Apicolytic Chondroflexing Thoracoplasty", a procedure that has proven to be effective, with no complications and without the long-feared deformation issues.

\section{Technique}

The incision and exposure are the same as for a standard thoracotomy, a periscapular J-shaped posterior skin incision with division of the latissimus dorsi and trapezius and rhomboid muscles is followed by retraction of the scapula. The fifth rib is extirpated and from that space the extrapleural detachment of the lung apex (apicolysispneumolysis) is carried out down to the aortic arch in the left side or to the azygos vein in the right side. Next, the fourth, third and second ribs are divided posteriorly as close to the transverse processes as possible. The intercostal bundles between the first and second ribs are sectioned along the upper border of the second rib. A small notch is made in the posterior end of the second rib. A corresponding small notch is made in the sixth rib as close to the vertebral column as possible. This entire osteomuscular flap is then bent down dorsally towards the most posterior side of the sixth rib as close to the mediastinum as possible.
Silk threads (gage \#2) are firmly tied (subperiosteally) into the notches. Thereafter, the lung apex is mobilized under the bent flap and sutured to the most lateral part of the sixth rib by applying gentle traction. The silk threads are then tightly drawn, and firmly tied when the bent flap forms a stable roof over the mobilized lung apex. The wound is closed in layers without drainage.

\section{Results}

Patients were operated on between 1987 and 2008; they were discharged upon their sputum cultures were negative and thereafter followed clinical and radiological surveillance.

Case 1: A 30-year-old woman with radiological and bacteriological diagnostic of PTB. After a 1-year treatment with isonicotinic acid hydrazide (Isoniazid), Ethambutol and Streptomycin, her cavernous lesion still persisted and was programmed for surgery (Figure 1A).

Case 2: A 32-year-old man, with radiological and bacteriological diagnostic of PTB that during his fourth month under medical treatment with Isoniazid, Ethambutol and Rifampicin, presented frequent episodes of hemoptysis requiring blood transfusions and therefore he was programmed for surgery (Figure 2A).

Case 3: A 35-year-old indigenous man, with radiological and bacteriological diagnostic of PTB with a two-year history of not adhering to treatment with Isoniazid, Ethambutol and Rifampicin, presented frequent severe cough episodes with hemoptoic sputum requiring hospitalization. The high risks of this patient of not adhering to treatment supported the decision to program him for surgery (Figure 3A).

Correspondence to: Alfonso Delgado, Facultad de Medicina y Ciencias Biomédicas, UACH, Circuito Universitario Campus II, Chihuahua, Chih, México, Tel: +52-(614)-4391846; Fax: +52 (614) 4391847; E-mail: aldelgado@uach.mx

Key words: tuberculosis, thoracoplasty

Received: August 14, 2017; Accepted: September 11, 2017; Published: September 14, 2017 

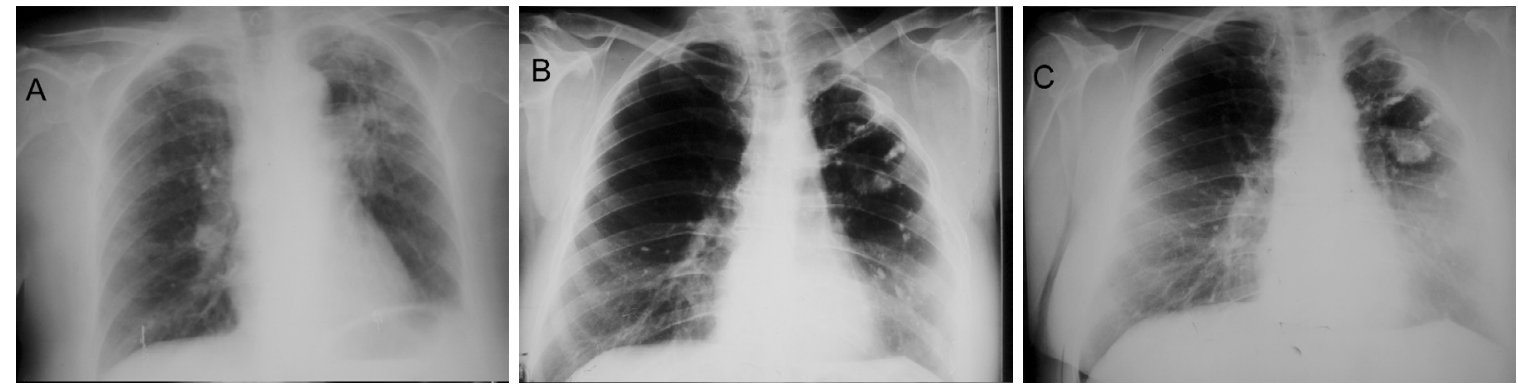

Figure 1. Chest x-ray films of case one. (A) Preoperative. A cavernous lesion can be observed in the left superior lobe; notice the presence of ipsi- and contralateral dissemination nodules. (B) Postoperative immediate. Shows a partial pulmonary collapse and a residual extra-thoracic air chamber. (C) Late postoperative, 22-years follow-up. Shows the cicatrized and calcified lesion; note also the calcified dissemination nodules on both lungs.
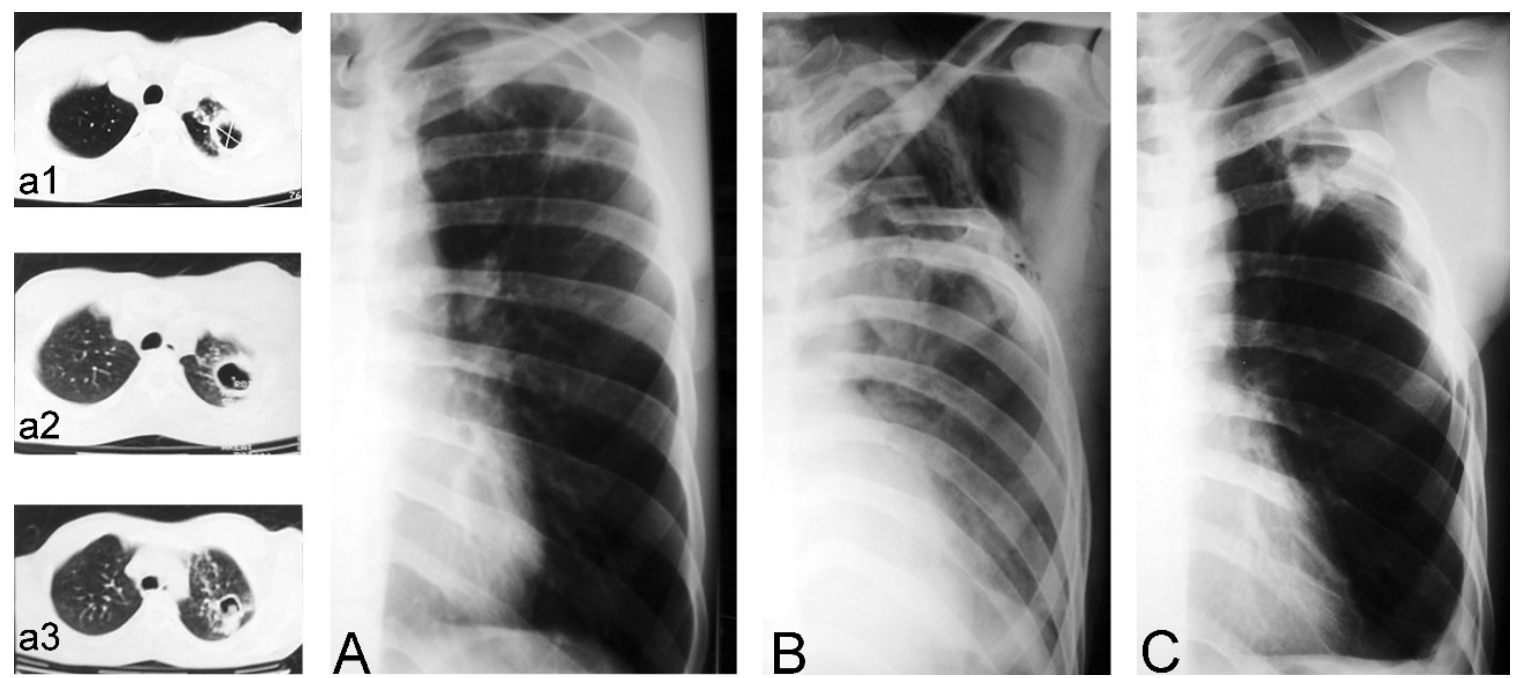

Figure 2. Chest x-ray films and thoracic computed tomographic (CT) scans of case two. (A) Shows a close-to-the-wall cavernous lesion in the left superior lobe. Lesion was confirmed by CT scans depicted in a1-a3. (B) Postoperative immediate. Shows a partial pulmonary collapse and a residual extra-thoracic air chamber. (C) Late postoperative, 10-years follow-up, showing the cicatrized and calcified lesion.
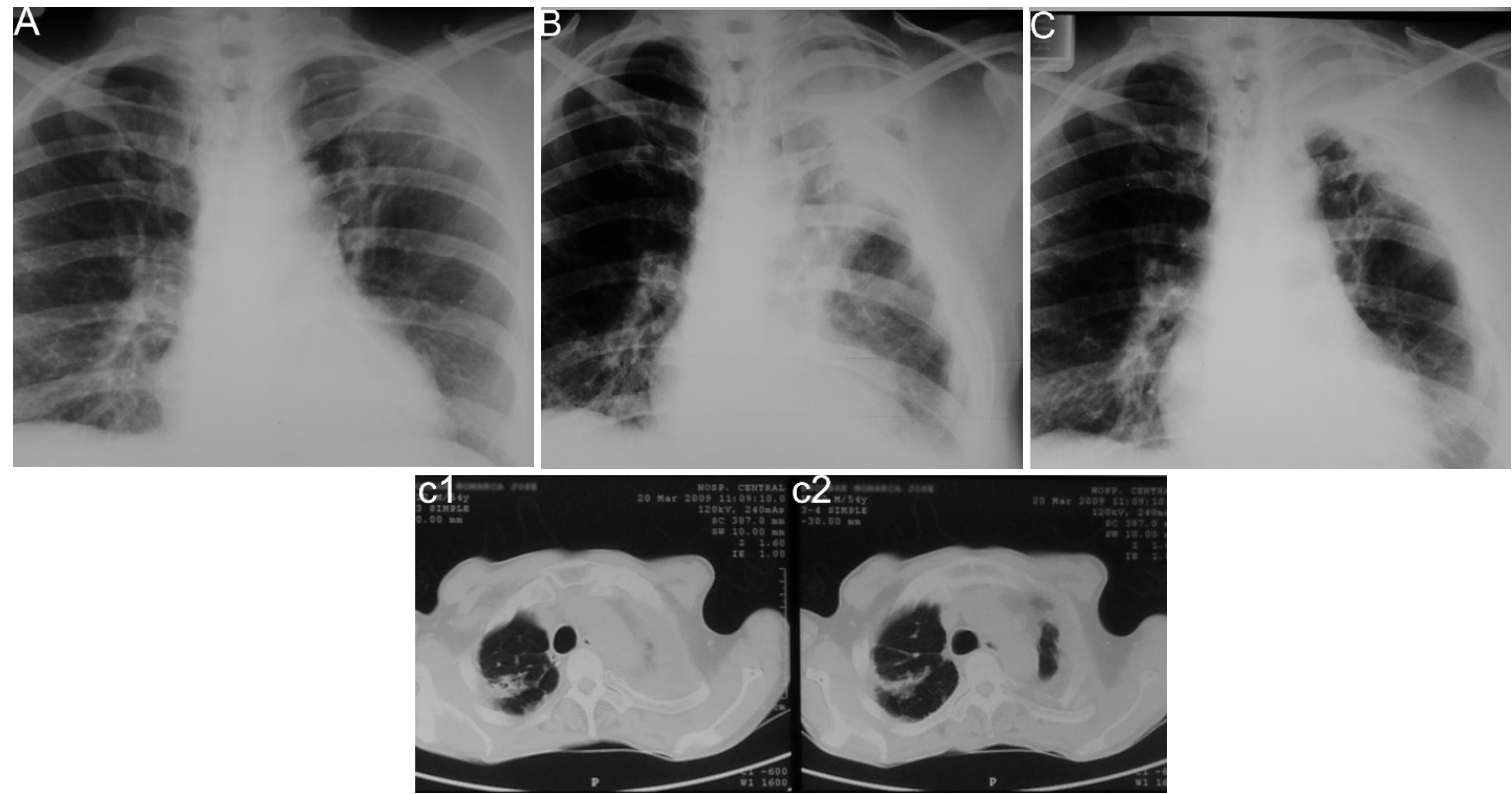

Figure 3. Chest $\mathrm{x}$-ray films and thoracic computed tomographic (CT) scans of case three. (A) Shows a thin-walled cavitated lesion in the left superior lobe. (B) Postoperative immediate. Shows a partial pulmonary collapse and a residual extra-thoracic air chamber. (C) Late postoperative, 2-years follow-up. A complete and stable collapse of the lesions is observed. Collapse was confirmed by CT scans shown in c1-c2. 


\section{Comment}

One of the main problems with cavernous lesions is that they are highly prone to hemorrhage and a source of tuberculous infection transmission. Collapse of these lesions greatly reduces the risk of hemoptysis and shortens the patient's infectious stage. Because the majority of this type of cavernous lesions is of apicodorsal location, we consider that the Apicolytic Chondroflexing Thoracoplasty is an effective surgical technique for PTB.

The containment of the post-operative re-expansion of the lung is the key to keep closed any cavernous process caused by tuberculosis. Bringing down the upper portion of the lung by the operative detachment of the parietal pleura allows the complete collapse of the pulmonary apex, and the chondrosternal flexion keeps the tuberculous cavity firmly collapsed without the need of anything else but the rigidity of the bent ribs (Figures 1B, 2B \& 3B), with the additional advantage of preserving the residual parenchyma.

Patient deformation was a chief concern for the indication and practice of thoracoplasty [1-2]. With the technique presented here such deformation is not an issue because the conservation of the first rib and the anterior and medium scalene muscles are left intact, avoiding the disfiguring cervico-thoracic scoliosis. In addition, it is remarkable that once the cavity is securely collapsed, the dissemination nodules spontaneously evolve to calcification (Figures 1C \& 2C). Moreover, these patients did not relapse during an over 20-year follow-up period.
In conclusion, the irreversible collapse of tuberculous caverns through the surgical technique presented here produces the cicatrization and calcification of cavernous lesions and dissemination nodules, without any ostensible anatomical deformation. Furthermore, the collapse of these lesions eliminates life-threatening hemoptysis and drastically shortens the time during which the patient is infectious. With proper selection of patients and early decision for surgical intervention, this technique can be a crucial adjunct for the treatment of complicated, treatment failure and MDR-PTB.

\section{References}

1. Einstein HE (2001) Out of the pages of history. Chest 120: 696-697. [Crossref]

2. Shepherd MP (1985) Plombage in the 1980s. Thorax 40: 328-340. [Crossref]

3. Horowitz MD, Otero M, Thurer RJ, Bolooki H (1992) Late complications of plombage. Ann Thorac Surg 53: 803-806. [Crossref]

4. Massard G, Thomas P, Barsotti P, Riera P, Giudicelli R, et al. (1997) Long-term complications of extraperiosteal plombage. Ann Thorac Surg 64: 220-224. [Crossref]

5. Weissberg D, Weissberg D (2001) Late complications of collapse therapy for pulmonary tuberculosis. Chest $120: 847-851$. [Crossref]

6. Martuzzi M, Valli A (1954) Anatomo-histological studies on apicolytic extrafascial supplemental cavity to thoracoplasty. Bull Sci Med (Bologna) 126: 25-37.

7. Barker WL (1994) Thoracoplasty. Chest Surg Clin N Am 4: 593-615. [Crossref]

8. Bjork VO (1954) Thoracoplasty, a new osteo plastic technique. J Thorac Surg 28: 194-211. [Crossref]

Copyright: (C2017 Salas A. This is an open-access article distributed under the terms of the Creative Commons Attribution License, which permits unrestricted use, distribution, and reproduction in any medium, provided the original author and source are credited. 